\title{
Development of the Korean version of ICF e-Learning tool
}

\author{
HaeJung Lee, JuMin Song \\ Department of Physical Therapy, College of Health and Welfare, Silla University, Busan, Korea
}

Purpose: The aim of the study was to develop a Korean version of an ICF e-Learning tool (KICF e-Learning tool).

Methods: The process of translation and adaptation of the ICF e-Learning tool was followed: two translators developed the Korean versions independently, and a consensus version of the translation was then produced. An expert committee, which was composed of five experts from physiotherapy, occupational therapy, speech pathology, and social welfare, reviewed the consensus Korean version to make a beta version of the tool. A field test was conducted to determine if the Korean version of the tool was easy to understand and suitable to use in ICF learning. Feedback from the field test were used for the final adaptation of the KICF e-Learning tool.

Results: One-hundred and twenty-six volunteers (40 males and 76 females) were invited to examine the KICF e-Learning tool. The participants reported various levels of ICF knowledge from none to very good. Forty-eight participants reported no knowledge of ICF. The majority of participants $(n=84)$ reported that Korean terms or expression in the tool were easy to understand and one-hundred fourteen participants would recommend the tool to another person. The Korean cases would be helpful for a Korean audience to study the ICF using the tool.

Conclusion: The KICF e-Learning tool was developed and is ready for use by the public for the consistency of ICF education. On the other hand, development of an advanced module will be needed.

Keywords: ICF e-Learning tool, ICF, education

\section{서 론}

기능수행(functioning)은 생리학적 기능과 해부학적 구조, 일상생활 의 수행능력 및 다양한 생활영역에서의 참여의 정도를 표현하는 포 괄적인 용어이다. 기능수행은 개인에게 영향을 주는 환경요인과 나 이, 성별, 건강상태 등을 포함하는 개인요인과 서로 상호 작용하며 개 인의 활동의 제한과 참여의 제약 정도를 나타낼 수 있다. 세계보건기 구(WHO)가 개발한 국제기능장애건강분류(ICF, international classification of functioning, disability and health, )는 건강과 관련된 기능수행 정도를 분류하는 국제분류체계로서 건강관련 개념을 다중적 관점 에서 접근할 수 있으며 다양한 전문영역에서 건강관련 정보의 의사 소통을 원활하게 한다. ICF는 보편성의 특성 즉 장애가 있는 사람을 포함하여 모든 개인에게 적용할 수 있다. 또한 삶의 질, 기능수행에 영 향을 주는 환경요인에 대한 평가, 교육과정 개발과 같은 연구 및 교육 에 사용되고 있다.

국내에서 ICF는 임상, 장애인, 특수교육, 지역사회, 노인, 체육학 등
다양한 영역에서 사용되고 있으며, 신체기능, 신체구조, 활동과 참여 및 환경요인을 나타내는 개념의 틀(framework), ICF 체크리스트, 특정 질환과 관련된 코어세트를 활용한 다양한 연구가 수행되었다. ${ }^{2}$

많은 연구에서 $\mathrm{ICF}$ 는 연구자의 연구목적에 맞게 기능수행과 관련 된 데이터의 수집 범위나 정도가 조정되어 개인의 기능수행 정도를 표현하였다. 이는 대상자 중심의 코드 선택이 아닌 연구중심의 코드 세트의 적용이라 할 수 있다. 활동 및 참여에 있어 편중된 데이터 수 집, 신체기능 및 구조 중심의 손상과 관련된 정보 위주의 데이터 수집 등은 기능수행에 대한 제한적인 프로파일링을 야기하여 개별적 기 능수행수준을 이해하는 데 어려움이 있었다. ${ }^{2-4}$ 이는 한 연구 간의 데 이터 비교가 어렵고, 이들 연구에서 수집된 데이터 또는 데이터 수집 방법은 관련 전문영역에서 보편화하여 활용하기에는 제한적이며, 또 한 정책이나 임상의 의사결정 활용에도 한계가 있다.

국내의 연구에서 특정 전문영역에서 사용목적에 따라 ICF항목을 선택하여 코어세트를 개발하는 것을 확인할 수 있었다. ${ }^{5,6}$ 이러한 결 과는 ICF코어세트개발을 위한 표준화절차에 따르지 않고 연구편의
Received Mar 18, 2019 Revised Apr 22, 2019

Accepted Apr 28, 2019

Corresponding author JuMin Song

E-mail jmsong@silla.ac.kr
Copylight (C2019 The Korean Society of Physical Therapy

This is an Open Access article distribute under the terms of the Creative Commons Attribution Non-commercial License (Http:// creativecommons.org/license/by-nc/4.O.) which permits unrestricted non-commercial use, distribution, and reproduction in any medium, provided the original work is properly cited. 
성에 의해 수행된 것으로 연구결과를 활용하는 데 제한이 있으며 이 는 ICF 특성인 보편성에 적합하지 않다. 또한 매년 개정되는 ICF 항목 을 적용하지 않는 연구도 많았다. 7. 국내의 ICF 관련 선행연구에서 확 인된 이러한 문제점들은 ICF에 대한 이해부족과 ICF활용방법의 오 류로 인한 것으로 판단된다. 이로 인해 ICF의 이해와 활용에 대한 표 준화된 교육의 필요성이 제안되고 있으나, 표준화된 교육과정이나 교 육 도구가 국내에는 부족한 실정이다.

보건의료전문가들은 업무의 특성상 임상 업무와 함께 주기적인 교육이 요구되지만 바쁜 일정으로 시간과 공간의 제약을 받는 전통 적인 방식의 교육은 한계가 있다. 이를 해결하기 위한 새로운 교육방 식이 요구되었으며 많은 학습자가 시간과 공간의 제약을 받지않고 자 기주도적으로 교육이 가능한 온라인학습도구의 개발이 보건의료전 문영역에서 보고되고 있다.9-11 선행연구에서 온라인 기반 학습도구를 사용한 교육이 보건의료전문가들이 지식 습득, 환자에 대한 태도 및 의사소통에서 향상이보고되었다. ${ }^{12}$

ICF에 대한 올바른 이해와 ICF 사용에 필요한 구체적인 지식을 접 근하기 쉽고 일관된 방식으로 가르치기 위해 $\mathrm{WHO}$ 에서 사용자가 쉽 게 접근할 수 있고 흥미롭게 학습할 수 있는 ICF 온라인 학습도구를 2018년에 개발하였다(https://www.icf-elearning.com/). WHO는 ICF의 올바른 사용과 발전을 위해서 각 회원국과 WHO-FIC 협력센터를 중 심으로 ICF 온라인학습도구의 번역을 권장하였으며, 이에 여러 국가 와 협력센터에서 번역작업이 진행되고 있다. ICF의 보급과 올바른 활 용을 위해 국내 사용자를 위한 표준교육도구로써 ICF 온라인학습도 구의 한글판 개발이 요구되며, 개발된 한글 ICF 온라인학습도구가 우 리나라의 문화와 관련 분야에서 사용하기에 적합한지 확인하기 위 한 필드테스트가 필요하다.

본 연구는 ICF의 국내 사용자를 위한 표준교육도구로서 활용하기 위해 ICF 온라인학습도구 한글본에 대한 필드테스트를 실시하고, 그 결과를 반영하여 ICF 온라인학습도구의 한글 최종본을 개발하기 위 해 수행되었다.

\section{연구 방법}

본 연구는 ICF 온라인학습도구의 한글번역, 번역본에 대한 전문가 검 토, 필드테스트를 위한 ICF 한글본(베타버전) 개발 및 필드테스트를 위한 설문지 개발의 순서로 진행되었다. 실시된 필드테스트의 결과 를 검토하고 반영하여 ICF 온라인학습도구의 최종 한글본을 도출하 였다.

\section{1. 대상자}

ICF 온라인학습도구 베타버전 개발을 위하여 5 명의 자문위원이 참
여하였다. 자문위원회는 물리치료, 작업치료 및 언어치료 분야에서 $\mathrm{ICF}$ 를 활용하고 교육하는 전문가로 구성되었다. 필드테스트는 ICF를 활용하거나 그 활용이 요구되는 물리치료, 작업치료, 재활의학, 보건 연구, 교육, 지역사회보건, 장애인단체를 포함한 전문영역의 전문가 및 학생을 대상으로 실시하였다. 모든 대상자는 본 연구의 목적과 내 용에 대한 설명을 듣고 연구에 참여할 것을 동의하였다.

\section{ICF 온라인학습도구 한글본(베타버전) 개발 및 필드테스트}

ICF 온라인학습도구는 용어사전, 탐색 안내, ICF에 대한 7개의 장 및 참고문헌으로 구성되어 있으며, 각 장의 주제는 ICF의 필요성, 목적, 사용, 모델, 구조와 코드, ICF 분류 척도 및 WHO-FIC (World Health Organization-Family of International Classifications, 세계보건기구-국 제분류군)에 대한 설명을 포함한다. 또한 각 장은 학습하기와 퀴즈의 영역으로 구성되어 있으며, 학습 능률을 향상시키기 위해 대화형 아 이콘을 포함하고 있다.

$\mathrm{ICF}$ 를 이해하고 사용하고 있는 보건의료 전문가 2 명이 ICF 온라인 학습도구를 독립적으로 번역한 후 비교 분석하여 합의본을 도출하 였다. 번역은 ICF 한글개정본의 용어를 기준으로 실시하였다.13,14 자 문위원은 ICF 온라인 교육도구 전체내용에 대한 한글용어 및 문맥상 의 한글표현을 검토하여 베타버전을 도출하였다. 또한 ICF 온라인학 습도구에 대한 평가를 위한 15 개의 문항을 검토하였으며, 한글번역 본에 대한 이해도와 만족도를 위한 3 개의 설문 문항을 개발하였다.

필드테스트를 위해 웹버전과 유사한 형식의 PPT 파일을 개발하였 다. PPT파일은 대화형식의 아이콘이 작동되도록 제작하였고, 각 장 의 퀴즈 및 필드테스트 설문은 구글 서베이를 통해 실시되었으며, 데 이터가 누적될 수 있도록 하였다. 필드테스트는 2018년 8월부터 11월 까지 약 4 개월간 실시되었다.

\section{3. 최종 한글판 개발}

필드테스트 설문결과를 반영하여 ICF 온라인학습도구 한글 최종본 을 개발하였다.

\section{4. 자료분석}

필드테스트 대상자의 특성과 설문결과는 IBM SPSS version 24.0 (SPSS $\mathrm{lcn}$, Chicago, IL)을 활용하여 기술통계로 분석하였다.

\section{결 과}

\section{ICF 온라인학습도구 한글본 베타버전}

베타버전은 필드테스트를 위해 PPT파일로 제작되었고 각 장의 퀴즈 와 필드테스트 설문은 구글서베이(http://bit.ly/FT_2018)를 통해 온라 

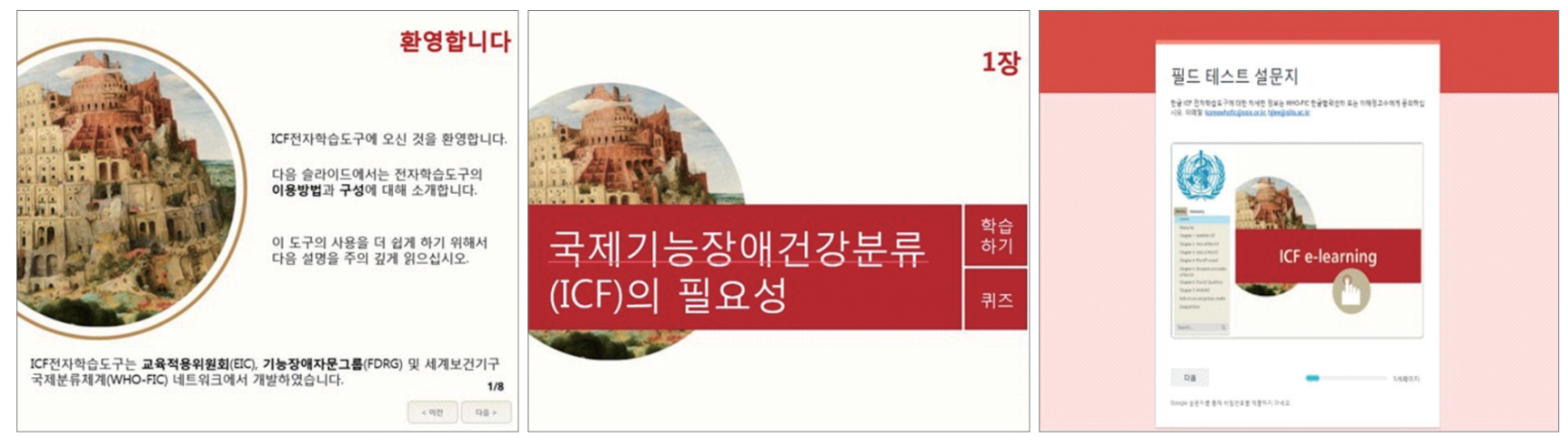

Figure 1. Examples of KICF e-Learning tool for the field test and online questionnaire.



Figure 2. Self-rated level of knowledge about ICF.

인상에서 실시되도록 하였다(Figure 1). ICF 온라인학습도구를 평가 하는 필드테스트 설문지문항에 추가하여 한글본의 이해도와 만족 도에 대한 설문항목 개발의 필요성이 제안되었으며, 개발된 3개의 문 항은 다음과같다.

16. ICF 온라인학습도구에서 개선해야 할 점에 대해 본인의 의견을 적어주세요

17. 한글 ICF 온라인학습도구에 사용된 한글용어와 한글표현을 이 해하는 데 어려움이 있습니까?

어려움 없음ㅁ 조금어려움 보통 어려움 매우어려움ㅁ

18. 한글 ICF 온라인학습도구에서 사용된 한글용어나 한글표현에 대해 수정이나 개선해야 할 부분이 있다면 구체적으로 적어주 세요.

\section{2. 필드테스트}

1) 대상자

필드테스트에 126 명(남 40 명, 여 86 명)이 참여하였으며, 평균나이 28.01세(19-58세)이다. 참여자들이 생각하는 본인의 ICF에 대한 지식 정도는 전혀 없음에서 충분한 지식이 있음으로 답하였다. 참여자 중 48 명은 지식 정도가 전혀 없음으로 답하였고, 나머지는 다양한 수준 의 지식 정도가 있는 것으로 답하였다(Figure 2).
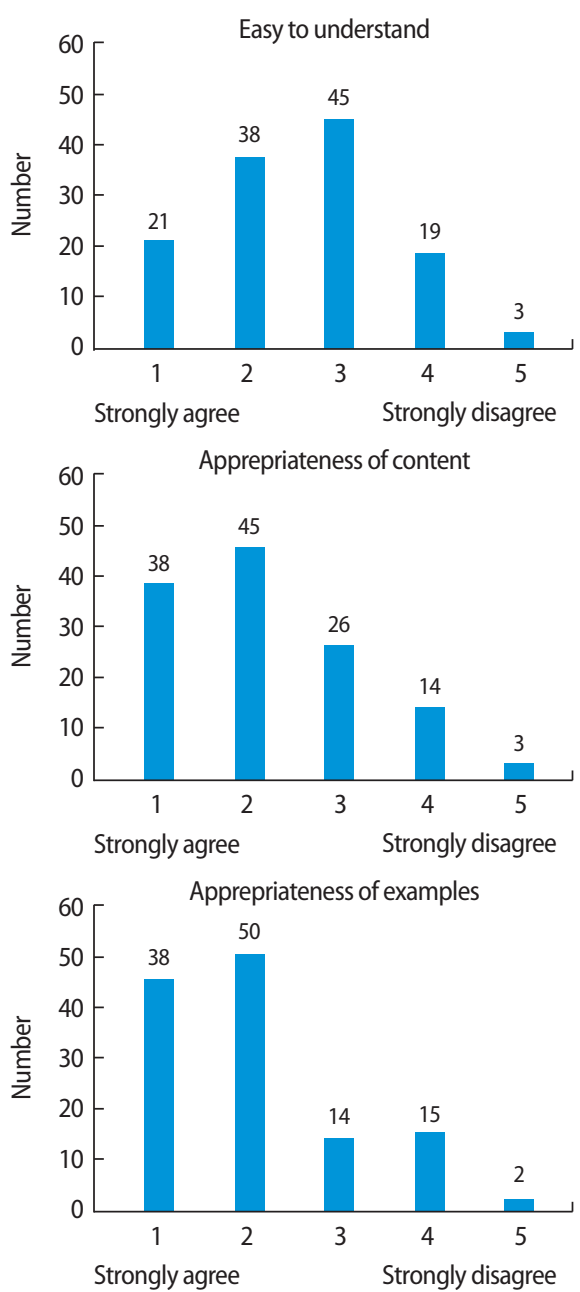

Figure 3. Response rates for contents of ICF e-Learning tool.

2) ICF 온라인학습도구의 내용에 대한 결과

참가자의 102 명 $(83 \%)$ 이 학습도구의 내용이 보통이상으로 이해하기 쉽다고 답하였다. 내용과 포함된 예시의 수준은 각각 105 명(86\%)의 참 가자들이 알맞은 수준(보통이상)이라고 응답하였다(Figure 3). 



Figure 4. Response rates for characteristics of ICF e-learning tool.

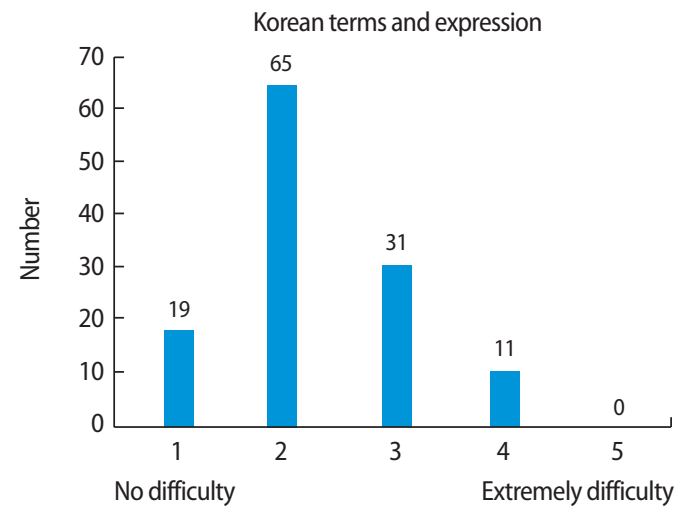

Figure 5. Understanding of Korean terms and expressions in KICF eLearning tool.

\section{3) ICF 온라인학습도구의 용이성에 대한 결과}

도구는 사용이 쉽고(101명, 82\%), 대화형식이며(96명, $7 \%$ ), 탐색기능이 직 관적(106명, 87\%)으로 잘 구성되어있다(보통이상)고 답하였다(Figure 4).

4) 한글용어와 표현에 대한 결과

한글용어와 표현의 이해 정도에 대한 문항에서 111 명(91\%)의 참여자 가 ICF 온라인학습도구의 내용이 보통이상의 수준으로 잘 이해된다 고 답하였다(Figure 5).

ICF 온라인학습도구(한글본)에서 한글용어나 표현에 대해 수정이 나 개선에 대한 의견을 분석한 결과, 내용, 예시, 도구 및 탐색기능으 로 분류하여 제시하였다. 내용에 대한 의견은 "한 페이지에 너무 많 은 내용이 포함됨, 중요한 부분에 대한 표시가 필요함, ICF 구조와 코 드와 분류척도에 대한 장에서 설명이 추가가 필요함” 등이 있었다. 현 재 도구에 포함된 예시에 대한 의견으로 "내용의 이해를 돕기 위한 예 시 또는 사례의 추가가 필요함, 예시에 대한 추가 설명이 요구됨” 등이 있었다. 각 장의 퀴즈에 대한 의견으로는 "퀴즈의 문항수의 추가가 필 요함, 난이도에 따른 퀴즈 문항의 개발이 필요함, 퀴즈 오답해설이 필 요함" 등이 있었다.
5) ICF 온라인학습도구에 대한 만족도에 대한 결과

ICF 온라인학습도구를 모두 학습하는 데 소요되는 시간은 대부분의 참여자가 90 분내외로 응답하였다. 또한 110 명의 참여자는 본 도구를 친구나 동료에게 추천하겠다고 응답하였다.

\section{4. 최종 한글판 개발}

필드테스트 설문결과를 반영하여 ICF 온라인학습도구 한글 최종본 을 개발하였다(https://www.icf-elearning.com/).

\section{고 찰}

본 연구는 ICF의 국내 사용자 교육을 위한 ICF 온라인학습도구의 한 글본 개발을 목적으로 126 명을 대상으로 필드테스트를 실시하였으 며 그 결과를 검토하고 반영하여 ICF 온라인학습도구의 최종 한글본 을 도출하기 위해 수행되었다. 대부분의 대상자들이 학습도구의 내 용이 이해하기 쉽다고 답하였다. 본 연구의 약 $30 \%$ 는 ICF에 대한 지식 이 없는 대상자들이 참여한 점을 고려한다면 ICF에 대한 기초교육도 구로서 적절한 것으로 판단된다. 국내 ICF 교육에 대한 선행연구와 교 육자료가 매우 부족하여 본 연구의 결과와 비교 분석하는 것은 제한 적이다.

개인의 기능수행정도를 표현하기 위해 ICF 전체 영역에 대한 자료 를 활용하는 것이 권장된다.1, 국내 임상영역에서 ICF를 활용한 사례 연구나 환자의 기능수행정도를 측정한 많은 선행 연구에 의하면 신 체 기능, 신체 구조 및 활동영역 중심으로 데이터를 수집하는 것이 관 찰되었다.15,16 이러한 참여와 환경 요인에 대한 데이터의 부재는 개인 의 기능수행정도를 프로파일링하는 데 제한적이다. ${ }^{17}$ 이는 전통적인 손상 중심의 의학적 모델에 근거한 임상 교육으로 기인된 것으로 사 료된다. 기능수행에 대한 생체심리사회적 모델이 발표된 것은 20 여년 이 지났으나 활용과 교육은 여전히 부족한 실정이다. 따라서 본 연구 결과로 도출한 ICF 온라인 학습 도구가 ICF에 대한 올바른 이해와 사 용에 도움이 될 것으로 기대한다.

상호작용을 위한 콘텐츠는 사례중심이나 문제중심으로 학습내용 
을 구성하며, 학습자의 참여를 유도하여 학습몰입도를 높인다. 이는 학습자의 지속적인 학습유지를 가능하게 하며 학습만족도와 성취 도를 높일 수 있다고 선행연구에서 보고되고 있다. ${ }^{2}$ 본 연구에서 ICF 온라인 학습도구를 구성하는 내용과 포함된 예시의 수준에 대해 대 다수의 대상자들이 ICF에 대해 학습하기에 적절한 수준으로 구성되 었다고 응답하였다. 그러나 사례와 사례에 대한 설명이 추가된다면 학습 도구의 내용을 좀 더 쉽게 이해하는 데 더 많은 도움이 될 수 있 을 것이라는 대상자들의 의견도 제시되었다. 이는 국내 실정에 맞는 사례와 설명이 강화된다면 국내 사용자의 ICF에 대한 이해를 향상시 키는 데 도움이 될 것으로 사료된다.

본 온라인학습도구에는 학습한 내용의 이해 정도를 확인하기 위 하여 각 장마다 퀴즈가 포함되어있다. 연구에 참여한 대상자들은 각 장에 포함된 퀴즈에 대한 의견으로 난이도가 다른 문항 개발, 문항수 의 추가, 오답해설의 필요성을 제시하였다. 일부 장에서 한 페이지에 너무 많은 내용이 포함되어 학습내용에 대한 가시성의 제한이 있으 므로 한 페이지에 포함되는 내용의 간소화가 필요하고, ICF 구조와 코드 및 분류척도에 대한 두 개의 장에서는 범위와 내용이 다소 어려 워 보완 설명이 추가된다면 ICF 온라인학습도구에 대한 학습자들의 이해도를 높이는 데 도움이 될 것이라는 제안도 있었다. 이러한 의견 은 WHO의 ICF 온라인학습도구 개발팀에 건의하여 향후 수정보완 작업에 반영할 수 있도록 제안하는 것이 필요할 것으로 판단된다.

대다수의 대상자들은 ICF 온라인학습도구가 직관적인 탐색기능 으로 구성되어 사용하기가 쉽다고 응답하였으며, 이는 본 학습도구 를 처음 접하는 학습자도 도구의 사용에 대해 직접적으로 파악하여 사용할 수 있는 것으로 사료된다. 본 도구의 대화형식의 구성은 전통 적인 대면방식의 교육의 장점인 학습자와 교육자의 상호작용을 대신 할 수 있는 구성으로 학습실재감을 유발하여 개인학습 참여에 효율 적일 것으로 기대한다.910 본 연구에서 개발된 한글본 ICF 온라인학습 도구는 사용만족도가 높은 것으로 확인되었다. 대부분의 본 연구 참 여자들은 ICF 온라인학습도구가 ICF를 이해하는 데 도움이 되며, 본 학습도구를 친구나 동료에게 추천하겠다고 응답하였다. 이는 온라인 학습도구의 만족도 조사에 대한 선행연구 결과와 유사하였다.12

본 도구의 번역에 사용된 한글용어 및 한글표현이 잘 이해되어 학 습하는 데 어려움이 없었다고 대부분의 대상자가 응답하였다. 이는 2016년에 개정된 ICF 한글본을 기준으로 하여 한글용어를 표준화하 였으며 ${ }^{13}$ 특정 전문영역의 용어가 아닌 일반인들도 이해하기 쉬운 용 어나 표현을 사용한 결과로 사료된다. 향후 ICF 관련 출판물이나 연 구에서도 표준화된 ICF 한글용어를 사용한다면 독자들의 이해에 도 움이 될 것으로 판단된다.

본 연구에서 개발된 한글본 ICF 온라인학습도구는 접근하기 쉽고 일관된 방식의 ICF에 대한 교육이 가능하고, 대화형식의 구성으로
학습자가 흥미롭게 학습이 가능할 것으로 사료된다. 본 도구는 ICF의 개발 목적과 이론적 근거에 기반한 ICF 이해와 바른 사용을 위한 교 육에 활용될 수 있을 것으로 기대되며, 국내 ICF 교육 표준화 기반을 조성하는 데 또한 기여할 것으로 생각된다. ICF에 대한 표준화된 교 육은 기능수행 및 장애에 대한 정보의 표준화에 기여하여 관련 데이 터를 질적으로 향상시키고 보건정책 개발 및 발전에 이바지할 것으 로 생각된다. 또한 한국실정에 근거한 사례의 개발, 다양한 전문영역 에 적합한 심화교육내용이 개발되어 다양한 전문영역에서 전문가들 의 ICF 교육에 사용된다면 전문가 사이의 의사소통에 기여할 것으로 생각되며 그 활용성이 확대될 것으로 판단된다. 향후 연구는 심화된 교육과정에 대한 콘텐츠개발과 ICF 교육 표준화에 대한 관리 및 모니 터링에 대한 연구가 필요할 것으로 생각된다. 또한 보건의료분야 특 성상 휴대와 정보 보관이 편리하고 오프라인 환경에서도 활용할 수 있는 전자책(e-book) 형식의 도구들이 사용되고 있다..$^{18}$ 전자책과 같 은 형식의 ICF 학습도구가 개발된다면 ICF의 이해와 바른 사용에 도 움이 될 것으로 사료된다.

\section{REFERENCES}

1. WHO. How to use the ICF: A practical manual for using the International Calssfication of Functioning disability and health (ICF): 0.9 version. Geneva, WHO, 2018.

2. Lee HJ, Song JM. An overview of the ICF's use in Korea. J Kor Phys Ther. 2015;27(5):356-61.

3. Lee HJ, Jumin S. Which information is commonly used for patients with stroke at rehabilitation settings? J Kor Phys Ther. 2015;27(6):392-99.

4. Song JM, Lee HJ. Investigating functional level in patients with stroke using ICF concept. J Kor Phys Ther. 2014;26(5):315-57.

5. Park M, Kang J. ICF core sets for measuring functional status of acute stroke patients. J Muscle Joint Health. 2012;19(2):107-19.

6. Kang J, Gong J, Park $\mathrm{M}$ et al. Assessing the functional status of acute stroke patients using ICF core set-short form. J Muscle Joint Health. 2013; 20(1):22-30.

7. Ahn D. Understanding of the international classification of functioning, disability, and health of WHO implications for leisure, recreation professionals and field of physical education in Korea. Kor Soc Study Phys Edu. 2013;18(3):15-24.

8. Kim C. How to conceptualize and measure disability in the employment policy-the comparison of common approaches and the application of ICF. J Vocat Rehab. 2011;20(3):138-99.

9. Warriner D, Bayle M, Shi Y et al. Computer model for the cardiovascular system: Development of an e-learning tool for teaching of medical students. BMC Med Edu. 2017;17:220-32.

10. Birns, B., Woodward P, Brenton H et al. Development of a novel multimedia e-learning tool for teaching the symptoms and signs of stroke. Sci Res. 2018;9(8):1196-211.

11. Bond SE, Crowther SP, Adhikari S et al. Design and implementation of a novel web-based e-learning tool for education of health professionals on 
the antibiotic vancomycin. J Med Internet Res. 2017;19(3):e93.

12. Moreira IC, Ramos I, Ventura S et al. Learner's perception, knowledge and behaviour assessment within a breast imaging e-learning course for radiographers. Eur J Rad. 2019;111:47-55.

13. WHO. International Classification of Functioning, Disability, and Health: Korean version. Seoul, Ministry of Health and Welfare, 2016.

14. Lee H, Song J. Development of revised Korean version of ICF. Kor Soc Phys Ther. 2014;26(5):344-50.

15. Kang T, No H. A case report of progressive intervention strategy applied
ICF tool about gait for TBI patient. J Kor Soc Phys Med. 2012;7(2):13747.

16. Kang T, Song M, Kim C. A case report of intervention strategy applied ICF tool on patient with low back pain. PNF and Movement. 2012; 10(2):17-24.

17. Lee H, Seo S, Song J. Information on functioning found in the medical records of patients with stroke. J Phys Ther Sci. 2016;28(10):2722-6.

18. Jang Y, Oh H. Investigating ebook use factors by medical students. J Kor Soc Infor Manag. 2014;2014(8):191-5. 